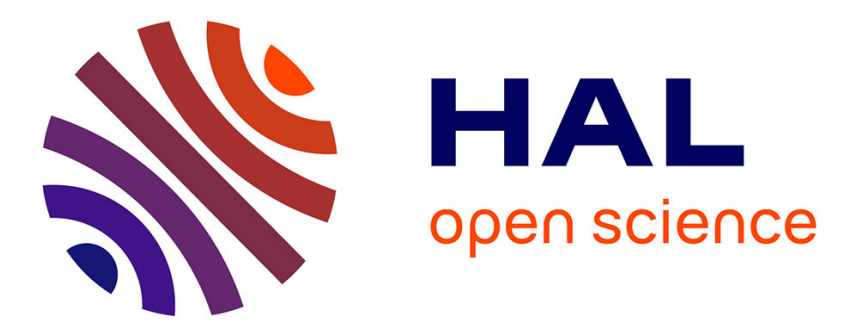

\title{
Sound propagation in liquid foams: Unraveling the balance between physical and chemical parameters
}

Juliette Pierre, Brice Giraudet, Patrick Chasle, Benjamin Dollet, Arnaud Saint-Jalmes

\section{- To cite this version:}

Juliette Pierre, Brice Giraudet, Patrick Chasle, Benjamin Dollet, Arnaud Saint-Jalmes. Sound propagation in liquid foams: Unraveling the balance between physical and chemical parameters. Physical Review E: Statistical, Nonlinear, and Soft Matter Physics, 2015, 91 (4), pp.042311. 10.1103/PhysRevE.91.042311. hal-01146098

\section{HAL Id: hal-01146098 \\ https://hal.science/hal-01146098}

Submitted on 27 Apr 2015

HAL is a multi-disciplinary open access archive for the deposit and dissemination of scientific research documents, whether they are published or not. The documents may come from teaching and research institutions in France or abroad, or from public or private research centers.
L'archive ouverte pluridisciplinaire HAL, est destinée au dépôt et à la diffusion de documents scientifiques de niveau recherche, publiés ou non, émanant des établissements d'enseignement et de recherche français ou étrangers, des laboratoires publics ou privés. 


\title{
Sound propagation in liquid foams: unraveling the balance between physical and chemical parameters
}

\author{
Juliette Pierre, ${ }^{*}$ Brice Giraudet, Patrick Chasle, Benjamin Dollet, and Arnaud Saint-Jalmes \\ Institut de Physique de Rennes, UMR 6251/Université de Rennes 1, Campus Beaulieu, 35042 Rennes Cedex, France
}

(Dated: April 27, 2015)

\begin{abstract}
We present experimental results on the propagation of an ultrasonic wave $(40 \mathrm{kHz})$ in liquid foams, as a function of the foam physical and chemical parameters. We have first implemented an original setup, using transducers in a transmission configuration. The foam coarsening was used to vary the bubble size (remaining in the sub-millimeter range), and we have made foams with various chemical formulations, to investigate the role of the chemicals at the bubble interfaces or in bulk. The results are compared with recently published theoretical works, and good agreement are found. In particular, for all the foams, we have evidenced two asymptotic limits, at small and large bubble size, connected by a non-trivial resonant behavior, associated to an effective negative density. These qualitative features are robust whatever the chemical formulation; we discuss the observed differences between the samples, in relation to the interfacial and bulk viscoelasticity. These results demonstrate the rich and complex acoustic behavior of foams. While the bubble size remain here always smaller than the sound wavelength, it turns out that one must go well beyond mean-field modeling to describe the foam acoustic properties.
\end{abstract}

\section{INTRODUCTION}

Aqueous foams are natural and self-organized hierarchical materials. They consist of packed gas bubbles separated by a continuous fluid network [1-3]. From the bubble point of view, there are no spatial arrangement, and the resulting material is amorphous. On the opposite, the liquid network surrounding the bubbles is selforganized with a well controlled geometrical structure [13]. To minimize the interfacial energy, which is proportional to the area of the gas-liquid interfaces, the fluid is finally distributed within films, liquid channels (called "Plateau borders" - PBs), and nodes of different geometries and sizes, and corresponding to minimal surfaces. The films are the liquid lamellae separating the bubbles, and are connected to the PBs, as the main elements of the network. These PBs are then connected to each others by the nodes. The important point is that the sizes are drastically different: films are flat lamellae of less than 1 micron thick, while Plateau borders have a pipe shape and have sections of characteristic length scale of typically tens to hundreds of microns. The nodes have the same characteristic size as the PBs, but are tetrahedral in shape. Most of the liquid is contained within the PBs and the nodes; though they contain a negligible amount of liquid, the films are crucial as their stability controls the whole foam stability.

The liquid fraction of the foam, $\Phi$, is defined as the volume of liquid divided by the volume of foam. A low liquid fraction implies a network with long and slender PBs, and films covering a large part of the bubble area. Oppositely, wet foams have small area of films between bubbles, as swollen PBs and nodes widely cover the bubbles. Back to the bubble point of view, the liquid fraction

\footnotetext{
* Present address: Institut Langevin, UMR 7587 CNRS, ESPCI, 75238 Paris Cedex 05, France. juliette.pierre@espci.fr
}

describes how packed the bubbles are. Together with the size of the bubble, the liquid fraction is a key parameter of a foam, as most of the foam properties depend on it. Moreover, the liquid fraction and the bubble size are not constant with time. Foams are out-of-equilibrium materials which drain and coarsen, resulting in a decrease of liquid fraction and increase of bubble size with time. Therefore, bubbles are getting more and more packed with time, as well as the liquid network gets emptied.

At the macroscopic scale, the foam can then be considered as a soft poroelastic material, as a result of the liquid and gas distribution at the scale of the bubble and liquid network. But, depending on its liquid fraction, bubble size, chemical formulation and external forcing, a foam can behave more like a liquid or a solid. Because of this wide and adjustable pallet of behaviors, foams have been chosen as the most efficient template in many applications [1, 3].

However, despite this wide use, many issues are still pending. First, techniques to characterize foams in details still have to be developed. In parallel to technical challenges lies a more general one, which is the propagation of waves in self-organized hierarchical and aging materials. In that spirit, the optics and the electrical transport of foams have been studied $[4,5]$. In a foam, light is scattered by the gas-liquid interfaces; with already a few bubble in thickness, the multiple scattering regime is obtained, and a foam is no longer transparent. This apparent disadvantage has been overcome, and in this limit of multiple scattering, many information can still be obtained [4, 6-8]. However, a major drawback with such multiple scattering is that one cannot directly see the internal organization within a foam.

By comparison, the acoustics of $3 \mathrm{D}$ foams has been much less studied. The key point is to understand how a soft liquid skeleton vibrates and eventually how such vibrations modify the propagation of sound from the one in a pure gas. Implementing earlier works [9-11], signifi- 
cant experimental and theoretical progress have recently been reported on foam acoustics [12-18]. Some of these were obtained with experimental setup based on ultrasonic transducers $[14,15,18]$. Other experiments with impedance tube allowed to broaden the range of investigated frequencies $[12,16]$. In parallel, light scattering techniques were used to monitor the acoustic deformation within a foam [13]. It turns our that these results can be rationalized by a global model, introducing resonance effects and a negative density regime [18]. In particular, these results emphasize the crucial role of the foam structure (bubbles size and liquid fraction).

Here, our objectives are twofold: first, we want to upgrade previous works performed with ultrasonic transducers [14], to improve the information which can be extracted by this approach. In particular, we want to monitor more precisely how the propagation depends on the bubble size, to further test the recent model of [18]. The goal is to determine if one can finally understand the continuous evolution of the acoustic features as the bubble size is widely varied. Secondly, we want to determine whether the chemical formulation can affect sound propagation. At this stage, it is not included in models, except in terms of a surface tension. However, unexpectedly high sound velocities were found for commercial foams $[10,16]$, possibly showing a possible role of chemistry.

To address these questions, we take advantage of the natural foam coarsening to vary the bubble size, starting from a controlled initial liquid fraction remains almost constant (between 8\% and 13\%). Furthermore, we have varied the chemical formulation in a controlled manner: our selection of solutions allow us to decorrelate as much as possible the effects occurring at the interfaces, in the films and in bulk. Thanks to these approaches, which are described in Secs. and III, we present new results, show that they agree with the recent theoretical predictions, and discuss the role of the physical and chemical parameters on foam acoustics.

\section{FOAM SAMPLES: CHOICE OF CHEMICAL FORMULATIONS AND GAS}

One of the goal of this work is to determine if the chemical formulation of the foam has an impact on the foam acoustics. Various structural and rheological properties are set by the chemical species of the foaming solution. First, they set the properties of the air-liquid interfaces. A first important quantity is the surface tension, decreased by the presence of adsorbed surfactants; but the adsorbed chemicals can also induce $2 \mathrm{D}$ viscoelasticity (either in compression or shear) [19, 20]. They can also modify the bulk properties of the solution, by providing non-Newtonian viscoelastic behavior [21]. More indirectly, the chemicals also control the properties of the films between two bubbles (thickness, spatial uniformity and viscoelasticity) [3, 22, 23]. Moreover, the viscoelastic behavior within a thin film separating two bubbles can be different from the one of the bulk, due to the confinement, especially for solutions containing polymers or proteins $[3,23]$.

Our plan was to select a reference formulation, then to add other components so that interfacial, film and bulk properties are tuned. This implied that we have selected chemical additives which modify, as much as possible, only a single property at a single scale (interface, film or bulk). In parallel, to quantify the modifications made by the additives in bulk, within the films and at the interfaces, we performed interfacial tensiometry, interfacial dilational rheology (with a pendant drop apparatus [19]), bulk viscoelasticity, and single film microscopy. For the latter, we used a home-made setup derived from the "thin film balance" apparatus to get information about the film texture and thickness [23, 24].

Following this strategy, we started with a simple and classical system: a solution of sodium dodecyl sulfate (SDS), a low molecular weight anionic surfactant. It was used here at $10 \mathrm{~g} / \mathrm{L}$, above the SDS critical micellar concentration (cmc). This surfactant solution has a high foamability, and corresponds to basically no interfacial viscoelasticity. The bulk solution viscosity is equal to the one of water and the films between the bubbles are uniform in texture, and in the order of tens of $\mathrm{nm}$.

A second group of formulations corresponds to the addition of dodecanol (DOH) to the SDS solutions. As the concentration of $\mathrm{DOH}$ is increased, the interfacial tension decreases, and an interfacial viscoelasticity emerges: the interfacial modulus increases as shown in Tab. I. Interestingly, this occurs while bulk and film properties remain constant. Thus, DOH is used to only tune the viscoelasticity of the gas-liquid interfaces. As a third choice, we have added glycerol to the SDS solution. With this additive, we can modify the bulk viscosity, with interfacial and film properties constant and similar to those of the SDS solutions.

To mimic more complex formulations, especially those found in industrial applications and commercial foams, we also performed experiments where a cationic polyelectrolyte $(\mathrm{CP})$ is added to the SDS solution. We have used a high molecular weight polymer, from Guar gum, known as Jaguar C13-S. At low concentration of CP $(0.2 \mathrm{~g} / \mathrm{L})$ added to the reference SDS solution, no effect are seen on the bulk and interfacial properties. When the concentration reaches a few $\mathrm{g} / \mathrm{L}$, various effects occur: the bulk viscosity is increased, the interfaces become rigid and the films becomes thicker and get gelified, as observed under the microscope. In fact, the interfacial rigidity is so high that no reliable measurements of the dilational viscoelastic modulus can be done with the pendant drop apparatus. We selected concentrations so that the bulk viscosity is the same as when glycerol is added, but the interfacial and films are also modified when the CP added.

Lastly, we also prepared solutions of milk proteins (casein); these proteins are widely used in food applications for making foams [25]. This allows us to get another complementary configuration: the bulk viscosity remains the 
one of water, while, due to confinement, the films between bubbles are heterogeneous, thick and gelified. Thus, here by comparing with SDS or SDS/DOH systems, only the films are modified.

The details of the interfacial and bulk measurements are given in the table 1 below. As a consequence of our choice of chemical systems, almost all types of combinations are tested here, and by comparing the results found for these solutions, one expects to decorrelate the possible influence of the interfaces, the films and the bulk.

For the gas, all the foams are made initially with hexafluoroethane, $\mathrm{C}_{2} \mathrm{~F}_{6}$. It is chosen to obtain a suitable coarsening rate of the foam: due to the its low solubility and diffusivity in water, the timescale for bubble size variation is much longer than for air or nitrogen, hence the timescale for a single acoustic measurement is also well shorter than the foam aging (see details below). The gas density $\rho_{g}$ and viscosity $\eta$ of this fluorinated gas are $\rho_{g}=5.84 \mathrm{~kg} / \mathrm{m}^{3}$ and $\eta=1.4 \times 10^{-5}$ Pa.s for $\mathrm{C}_{2} \mathrm{~F}_{6}$ (for comparison, $\rho_{g}=1.4 \mathrm{~kg} / \mathrm{m}^{3}$ and $\eta=1.8 \times 10^{-5}$ Pa.s for air).

It is important to note that at their creation, foams are made with pure $\mathrm{C}_{2} \mathrm{~F}_{6}$, but air tends to enter and diffuse inside the foam, leading to a volumetric expansion of the foam [26]. Therefore, the composition of the gas changed as a function of time. In the following data analysis, we discuss in details the effect of this change of gas composition with time.

\section{FOAM SAMPLES: PRODUCTION AND PHYSICAL PARAMETERS}

All the foams tested here are produced using the double syringe technique, illustrated in [16]. For all the chemical formulations, the liquid fraction $\Phi$, just after production at $t_{0}$ is $0.10 \pm 0.02$. The bubble radius is systematically measured during the acoustical measurements and all the initial mean bubble radii are found lower than 70 microns. We have chosen such initial conditions to have foams which mostly age by coarsening [27]; this is due to the small bubble radii, which induces high pressure in bubbles, and high coarsening rate, while drainage rates are oppositely low. Moreover, we used samples of a few $\mathrm{cm}$ high; this also reduce the drainage as our samples have a height comparable to the capillary hold-up distance [28], which corresponds to the part of a foam which always remain imbibed of water at the bottom of a foam.

To monitor the bubble size evolution, we use the "bubble raft method", meaning that a tiny portion of a foam is collected over time and deposited on a bath of soap solution (typically the foaming solution of SDS at $10 \mathrm{~g} / \mathrm{L}$ ) in order to get a raft [16]. From image analysis, the bubble size distribution, $n(r)$, is found as a function of time. It can be fitted by a log-normal law:

$$
n(r)=\frac{1}{R \varepsilon \sqrt{2 \pi}} \exp \left(-\frac{1}{2 \varepsilon^{2}} \ln ^{2} \frac{r}{R}\right)
$$

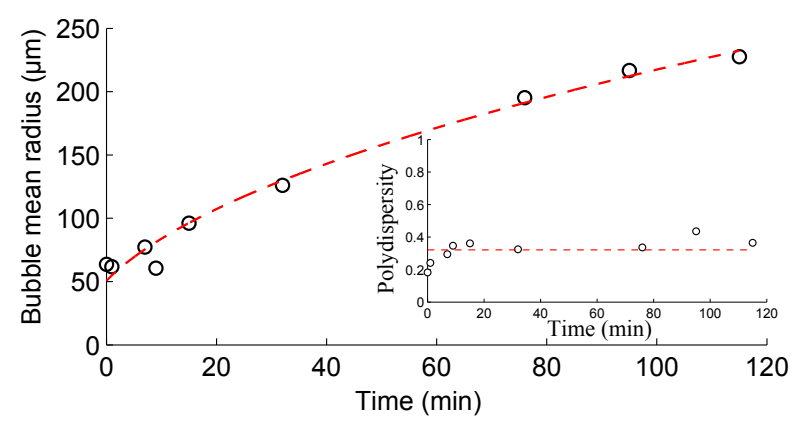

FIG. 1. (Color online) Time evolution of the mean bubble radius of a pure SDS foam with $\mathrm{C}_{2} \mathrm{~F}_{6}$ placed in open air. The curve is a fit with the evolution law (2) with $r_{0}=51 \mu \mathrm{m}$, $t c=344 \mathrm{~s}$. Inset: time evolution of the polydispersity.

where $r$ is the bubble radius, $R$ the median radius and $\varepsilon$ the polydispersity. The mean radius is $\langle r\rangle=R \mathrm{e}^{\varepsilon^{2} / 2}$. The parameters $\langle r\rangle$ and $\varepsilon$ are measured as a function of time for all the chemical formulations.

An example is given in Fig. 1. These are data for the SDS reference foam. The average bubble radius, $\langle r\rangle$, follows the usually reported law $[4,7]$ :

$$
\langle r\rangle=r_{0} \sqrt{1+\frac{t}{t_{c}}}
$$

where $r_{0}$ is the initial average bubble radius and $t_{c}$ a characteristic time, which depends on the chemical formulation. Note that the polydispersity does not strongly depend on time. In the following, such relationship between bubble size and time is measured for all the different types of foams, and is used to transpose the acoustic data from a time-dependance to a size dependance.

Concerning the liquid fraction $\Phi$ and its evolution with time, we previously monitored its evolution for SDS foams in similar geometries [14], and have shown that it varied by less than a factor two over the experimental timescale. Together with the facts that we used here small initial bubbles and small sample heights, drainage can be considered as a minor effect when compared to coarsening. However, we must also take into account that air is progressively invading the foam, and this tends to decrease the overall liquid fraction, as the foam volume increases. This liquid fraction evolution is also discussed as we analyze the acoustic data shown below.

\section{ULTRASONIC MEASUREMENTS}

The other goal of this work is to upgrade the experimental ultrasonic setup previously described in [14], who observed dispersion in coarsening SDS and Gillette foams, and a minimum of transmission at a given time, ascribed to resonance effects.

In the experiments presented in [14], the foam was placed in open air between two fixed piezoelectric trans- 
TABLE I. Table of physico-chemical properties of the different foaming solutions. The interfacial dilatational moduli were measured at $1 \mathrm{~Hz}$ and amplitude $\delta A / A_{0}=0.05 \%$; symbols $\gg$ stand for the solutions where they were too high to be measured reliably.

\begin{tabular}{llll}
\hline Foaming solution & surface tension $(\mathrm{mN} / \mathrm{m})$ & Interfacial modulus $E(\mathrm{mN} / \mathrm{m})$ & Bulk viscosity $(\mathrm{mPa} . \mathrm{s})$ \\
\hline SDS $(10 \mathrm{~g} / \mathrm{L})$ & 36 & 0 & 1 \\
SDS $(10 \mathrm{~g} / \mathrm{L})+$ DOH $(0.03 \mathrm{~g} / \mathrm{L})$ & 31 & 3 & 1 \\
SDS $(10 \mathrm{~g} / \mathrm{L})+$ DOH $(0.1 \mathrm{~g} / \mathrm{L})$ & 24 & 11 & 1 \\
SDS $(10 \mathrm{~g} / \mathrm{L})+$ DOH $(1 \mathrm{~g} / \mathrm{L})$ & 19 & 33 & 1 \\
SDS $(10 \mathrm{~g} / \mathrm{L})+$ CP $(0.2 \mathrm{~g} / \mathrm{L})$ & 36 & 0 & 10 \\
SDS $(10 \mathrm{~g} / \mathrm{L})+$ CP $(3 \mathrm{~g} / \mathrm{L})$ & 36 & $\gg$ & 16 \\
SDS $(10 \mathrm{~g} / \mathrm{L})+$ CP $(4 \mathrm{~g} / \mathrm{L})$ & 36 & $\gg$ & 7 \\
SDS $(10 \mathrm{~g} / \mathrm{L})+$ Glycerol $(4 \mathrm{~g} / \mathrm{L})$ & 36 & 0 & 16 \\
SDS $(10 \mathrm{~g} / \mathrm{L})+$ Glycerol $(7 \mathrm{~g} / \mathrm{L})$ & 36 & 0 & 1 \\
Casein $(5 \%)$ & 48 & 20 & 1 \\
Casein $(10 \%)$ & 50 & 22 & 1 \\
\hline
\end{tabular}

ducers working at $40 \mathrm{kHz}(\mathrm{MA} 40 \mathrm{E} 7 \mathrm{R} / \mathrm{S})$ at a fixed distance. The complex transmission at one distance and one frequency $(40 \mathrm{kHz})$ was followed during foam aging. The complex transmission is given by the amplitude and the phase of the received signal, measured by an oscilloscope. Several experiments, each one at a different, given distance, were performed to measure the speed of sound and attenuation.

In such a setup, neglecting multiple interferences, the complex transmitted signal $T$, in between the two transducers separated by a distance $x_{i}$, writes:

$$
T=|T| \exp (\mathrm{i} \varphi)=\frac{4 Z Z_{t}}{\left(Z+Z_{t}\right)^{2}} \exp \left(i x_{i} k\right)
$$

where $Z$ and $Z_{t}$ are respectively the complex impedance of the foam and the transducers, and $k$ is the complex acoustic wave vector, which can be written as:

$$
k=\frac{2 \pi f}{v}+\mathrm{i} \alpha .
$$

Hence, the logarithm of the absolute value of the transmission is linked with the sound attenuation by the linear relation:

$$
\ln (|T|)=-\operatorname{Im}(k) x_{i}+\operatorname{Re}[A]=-\alpha x_{i}+\operatorname{Re}[A],
$$

and the phase of the transmission is linked with the phase velocity by the linear relation:

$$
\varphi=\operatorname{Re}(k) x_{i}+\operatorname{Im}[A]=\frac{2 \pi f}{v} x_{i}+\operatorname{Im}[A],
$$

where:

$$
A=\ln \frac{4 Z Z_{t}}{\left(Z+Z_{t}\right)^{2}} .
$$

Therefore, obtaining the phase velocity and the attenuation through the foam is in principle possible by linear fits from a set of experiments done at different distances (a)

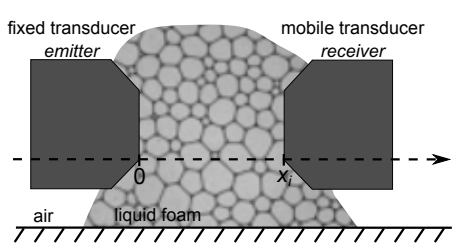

(b)

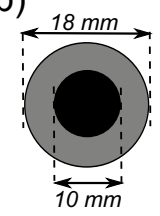

(c)
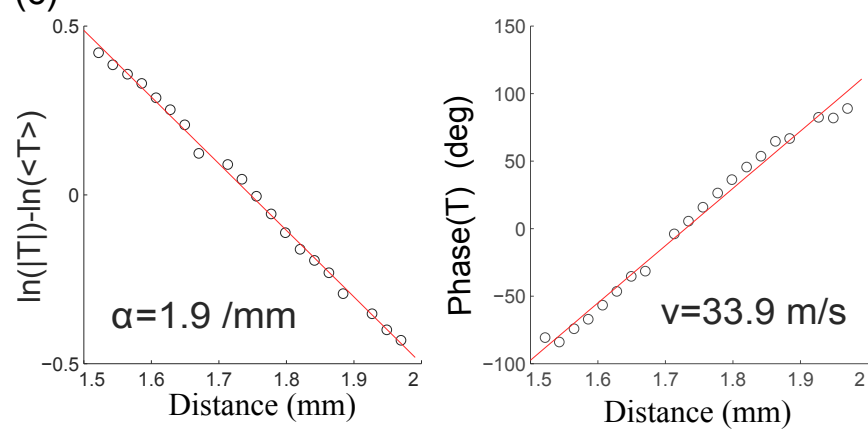

FIG. 2. (Color online) (a) Sketch of the setup. (b) Dimensions of the transducers. (c) Data analysis: plots of the logarithm of the transmission $\ln |T|$ and of the phase as functions of the distance, on one single translation of the receiving transducer. The logarithm of the average transmission, $\ln \langle T\rangle$, is subtracted to $\ln |T|$ only for convenience; this has no effect on the slope of the fit (hence on the value of $\alpha$ ). The best linear fits are shown, which give the attenuation coefficient and the speed of sound.

between transducers. However, this methods crucially relies on a reproducible evolution of the samples used in different experiments, and it turned out that the reproducibility of the coarsening of the different samples was not good enough to measure reliably the speed of sound and the attenuation.

To overcome this difficult, we need to vary the distance on a given sample. Hence, in our new setup, the liquid foam is still placed in the open air in between the couple of narrow-band transducers working around $40 \mathrm{kHz}$ 
(Fig. 2.a and b). But while the emitting transducer is fixed, the receiving transducer is mounted on a translation stage. This enables to vary the distance between the transducers $\left(x_{-}<x_{i}<x_{+}\right)$, hence to measure the velocity and attenuation in the foam on a path.

Figure $2 \mathrm{c}$ shows the phase and the absolute value of the complex transmission during one translation, for values of $x_{i}$ increasing between $1.5 \mathrm{~mm}$ and $2 \mathrm{~mm}$. This translation lasted less than 30 seconds. We can safely neglect foam coarsening at this time scale, this hypothesis being supported by the linear evolution of the phase of the transmission and the logarithm of the absolute value of the transmission in function of the distance. Looking for equations (6) and (5), the phase velocity $v$ and the attenuation $\alpha$ are estimated using a linear fit in which the phase is unwrapped prior to its fit. The uncertainty on this fitting procedure comes from the dispersion of the experimental data off the linear trend (Fig. 2c) and the $99 \%$ confidence interval criterion gives the error bars on $v$ and $\alpha$ shown later in Figs. 4 and 5 . We have not attempted to calibrate the absolute value of the phase, because it is not necessary to measure $v$. Hence, the phase shown in Fig. 2c, and later in Fig. 3, is true except for a (constant) offset. To improve the sensitivity of the ultrasonic setup, a lock-in has been also adding to the receiver. To validate the setup, we have checked that monitoring the dependence of phase on distance for transducers placed in air, we recover the speed of sound in air within $3 \%$.

Our experiments are thus performed as follows. A freshly prepared foam sample is injected between the two transducers. Then, the translation stage is set to make a continuous back-and-forth motion between distances $x_{-}$ and $x_{+}$, at constant velocity. In order to minimize the disturbance of the foam by the motion of the receiver, the amplitude of the translation $x_{+}-x_{-}$was kept below $2 \mathrm{~mm}$, although it should remain large enough that the amplitude and the phase vary significantly, to permit fitting by (5) and (6). We have checked that our procedure does not induce foam coalescence. We have also checked that the duration of a single translation was always much shorter than the typical coarsening time; hence, over a single translation, we probe a foam of nearly constant properties. As shown below, we point out that during the foam aging, the variation of the acoustic properties can be sufficiently strong, so that we need to change the starting and ending position to optimize the measurements.

\section{EXISTING MODELS}

As a key assumption to all models of foam acoustics, the bubble size is assumed to be much smaller than the acoustic wavelength $\lambda$, an assumption which is fulfilled in practice: the bubble size remains below $250 \mu \mathrm{m}$, whereas $\lambda=v / f$ is of order $1 \mathrm{~mm}$ to $1 \mathrm{~cm}$.

We will compare our measurements to the model of [18]. It is based on the vibrational response to pressure waves of the foam idealized as a 1D assembly of gas pockets separated by thin, flexible soap films of thickness $e$ and diameter $a$, connected to thick, rigid Plateau borders. The prediction of the acoustic wavevector is:

$$
k^{2}=\omega^{2} \chi_{\mathrm{eff}} \rho_{\mathrm{eff}},
$$

with $\chi_{\mathrm{eff}}=(1-\Phi) \chi_{g}+\Phi \chi_{w}$, and:

$$
\rho_{\mathrm{eff}}=(1-\Phi) \rho_{g}+\Phi^{\prime} \rho
$$

with an effective liquid fraction given by:

$$
\Phi^{\prime}=\frac{\Phi_{c}+\Phi_{f}(1-i \omega \tau) \mathcal{I}(q a)}{1+\left(x^{2} \frac{\Phi_{f}+\Phi_{c}}{\Phi_{f}}-2 x\right)[1-\mathcal{I}(q a)]-i \omega \tau x \mathcal{I}(q a)} .
$$

Let us describe the various terms in this expression. The liquid fraction of the foam $\Phi$ is separated in two contributions: $\Phi=\Phi_{f}+\Phi_{c}$, with $\Phi_{f}$ the contribution of the films and $\Phi_{c}$ that of the Plateau borders and vertices. The volume fraction of the films is $\Phi_{f}=\pi a^{2} e n_{f}$, with $n_{f}$ the number per unit volume of films perpendicular to the direction of propagation. Considering that there are $N$ such films per bubble, one obtains $n_{f}=3 N(1-\Phi) / 4 \pi r^{3}$. The volume fraction in the Plateau borders and vertices is $\Phi_{c}=\Phi-\Phi_{f}$. The characteristic time $\tau$ is a fitting parameter which describes dissipation phenomenologically. The quantity $q=\omega \sqrt{\rho e / 2 \sigma}$ is the characteristic wavenumber of the films. The dimensionless number $q a$ is the argument of the function $\mathcal{I}$, defined as:

$$
\mathcal{I}(q a)=\frac{\int_{0}^{\infty} a^{\prime 2} n\left(a^{\prime}\right) \mathcal{H}\left(q a^{\prime}\right) \mathrm{d} a^{\prime}}{\int_{0}^{\infty} a^{\prime 2} n\left(a^{\prime}\right) \mathrm{d} a^{\prime}}
$$

In this definition, $n\left(a^{\prime}\right)$ is the distribution function of the film radii $a^{\prime}$, assumed to follow a log-normal distribution like the bubble radii, with a polydispersity $\varepsilon_{f}$, and $\mathcal{H}$ is defined as:

$$
\mathcal{H}\left(q a^{\prime}\right)=\frac{2 J_{1}\left(q a^{\prime}\right)}{q a^{\prime} J_{0}\left(q a^{\prime}\right)}
$$

with $J_{0}$ and $J_{1}$ Bessel functions of the first kind. Hence, $\mathcal{I}$ is a weighted average of the function $\mathcal{H}$, whereby the foam polydispersity is taken into account in the model. Finally, $x$ is the mean surface fraction of the thin soap films on the bubble faces, the remainder being occupied by Plateau borders and vertices. The surface fraction covered by films in a liquid foam was studied by Princen [29]. He found the following empirical dependence on $\Phi$ : $x=1-3.20[7.70+(1-\Phi) / \Phi]^{-1 / 2}$.

A crucial ingredient of the model of [18] is that it accounts for the different response of the soap films and of the Plateau borders as the mean bubble radius and the frequency vary. Because of their difference in inertia, films and Plateau borders display the same vibrational response to pressure waves only below a critical 
frequency, which decreases at increasing bubble size. On the contrary, at large bubble size and frequency, the inertia of Plateau borders and vertices is such that their vibration can be neglected with respect to the films. As a consequence, the model of [18] naturally includes two models previously proposed: Wood's model [30], and Kann's model [11], as two distinct asymptotic limits, respectively of small bubble size and/or frequency, and of large bubble size and/or frequency. Let us remind these two models. Wood's model is an effective medium approach, and considers that the sound propagation is only sensitive to average properties of the foam, namely its average density and compressibility, irrespective of any microstructural or physicochemical detail. With such assumptions, Wood's law predicts a phase velocity given by the relation: $v_{\text {Wood }}=\left(\rho_{\text {Wood }} \chi_{\text {eff }}\right)^{-1 / 2}$, where $\rho_{\text {Wood }}=\Phi \rho+(1-\Phi) \rho_{g}$ is the foam density in its usual sense, and $\chi_{\mathrm{eff}}=\Phi \chi_{w}+(1-\Phi) \chi_{g}$ as previously defined. In these expressions, $\rho$ and $\rho_{g}$ are respectively the density of liquid and gas, and $\chi_{w}$ and $\chi_{g}$ the corresponding compressibilities. Since $\lim _{x \rightarrow 0} \mathcal{I}(x)=1$, it is straightforward to show from (10) that when $q a \rightarrow 0$, $\Phi^{\prime} \rightarrow \Phi$, hence from $(9), \rho_{\text {eff }} \rightarrow \rho_{\text {Wood }}$. Since typically $\chi_{w} / \chi_{g} \approx 10^{-4}, \chi_{\mathrm{eff}} \simeq(1-\Phi) \chi_{g}$. Hence, with $v_{g}=\left(\rho_{g} \chi_{g}\right)^{-1 / 2}$ for the speed of sound in gas, Wood's model yields:

$$
v_{\text {Wood }}=\frac{v_{g}}{\sqrt{(1-\Phi)\left(1-\Phi+\frac{\rho \Phi}{\rho_{g}}\right)}} .
$$

In the following, we will use the predictions (8) and (10) at low bubble size, when the acoustic behavior starts departing from Wood's law. More precisely, we perform a series expansion of the model at $q a \ll 1$. Using the expansions: $\mathcal{H}\left(q a^{\prime}\right) \simeq 1+q^{2} a^{\prime 2} / 8$ and $\mathcal{I}(q a) \simeq 1+q^{2} a^{2} \mathrm{e}^{5 \varepsilon^{2}} / 8$, after the moments of the log-normal law (1): $\int_{0}^{\infty} a^{\prime p} n\left(a^{\prime}\right) \mathrm{d} a^{\prime}=a_{0}^{p} \mathrm{e}^{p^{2} \varepsilon^{2} / 2}$. Assuming $\Phi_{f} \ll \Phi_{c}$, which is valid in practice, the effective liquid fraction (10) becomes: $\Phi^{\prime} \simeq \Phi /\left[1-\left(r / R^{*}\right)^{3}-\mathrm{i} \delta\right]$, with a damping coefficient $\delta=\omega \tau x$ and a resonance radius:

$$
R^{*}=\left[\frac{12 N \sigma(1-\Phi)}{x^{2} \rho \Phi \omega^{2} \exp \left(9 \varepsilon^{2} / 2+4 \varepsilon_{f}^{2}\right)}\right]^{1 / 3} .
$$

In Eq. (16), $\varepsilon_{f}$ is the polydispersity of the films, which is considered close to $\varepsilon$; see also the Supplemental Material of [18]. Inserting in the expression (8) of the acoustic wavevector and extracting the speed of sound from $v=\omega / \operatorname{Re}(k)$, we then get:

$$
v(R)=v_{\text {low }}\left\{\frac{1}{2}\left[1-\frac{R^{3}}{R^{\dagger 3}}+\sqrt{\left(1-\frac{R^{3}}{R^{\dagger 3}}\right)^{2}+\delta^{2}}\right]\right\}^{-1 / 2} \sqrt{\left(1-\frac{R^{3}}{R^{\dagger 3}}\right)^{2}+\delta^{2}},
$$

a formula valid for $q a \ll 1$. Following the theoretical derivation, $R^{\dagger}$ in Eq. (17) is equal to $R^{*}$. However, we distinguish $R^{*}$ and $R^{\dagger}$ because there are two ways of estimating the resonance radius: either by direct computation of (16), or by fitting with (17) the data at small bubble radius to get $R^{\dagger}$ as a best fit parameter; the two methods will be used in Sec. VII.

\section{RESULTS FOR THE REFERENCE FOAM}

water fluid phase containing SDS (10 g/L) and the gas

We first focus on a foam made with the simplest formulation: as discussed previously, this corresponds to a 

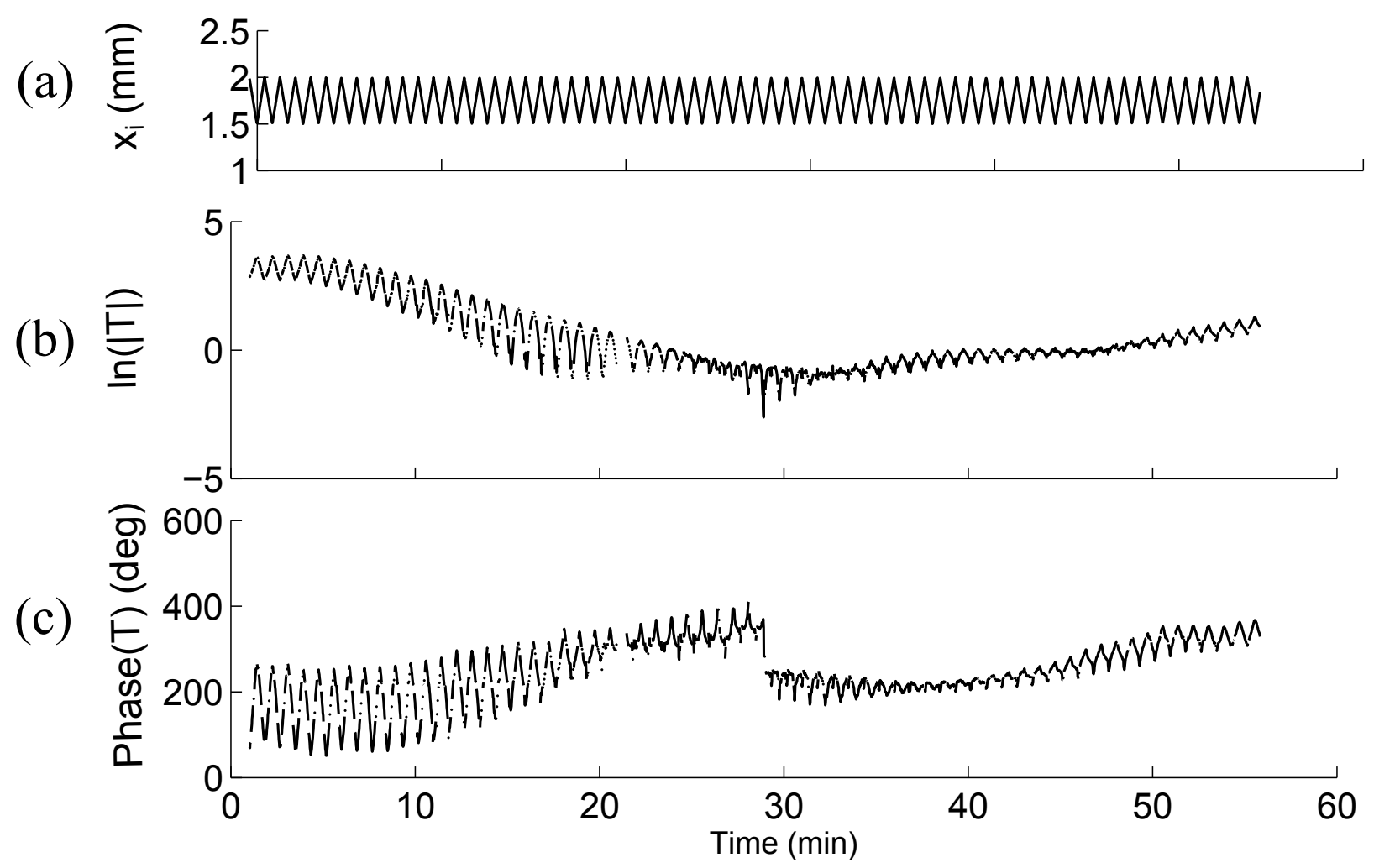

FIG. 3. Time evolutions of (a) the distance between the transducers, (b) the logarithm of the transmission, and (c) the phase of the transmission.

phase is $\mathrm{C}_{2} \mathrm{~F}_{6}$. With this solution, we present below in details all the acoustic features we can understand and extract by our measurement technique. Then, we discuss in the following section how these quantities depend on the physical chemistry.

Figure 3 shows the time evolution of the complex transmission through the SDS-foam. The distance between the transducers is varied between $x_{-}=1.5 \mathrm{~mm}$ and $x_{+}=2 \mathrm{~mm}$ up to 57 minutes (Fig. 3). For this foam, typically after 60 minutes of evolution, the boundaries $x_{-}$and $x_{+}$are no longer optimal: first, the variations of the transmitted signal along a path, become increasingly smaller with time, so that extracting the acoustic properties by the fitting procedure explained in Sec. IV becomes impossible. Moreover, as shown below, the range of bubble size corresponding to the resonance is actually passed after 60 minutes: it then becomes more interesting to collect data at much higher bubble sizes to cover the widest range of size variations. As a consequence, a second configuration with larger $x_{-}, x_{+}$and $x_{+}-x_{-}$is required. In practice, these distances have also to be adjusted to avoid too large deformation or destruction of the foam sample. In the case of the SDS foams, the values $x_{-}=6 \mathrm{~mm}$ and $x_{+}=8 \mathrm{~mm}$ corresponds to the best distances to get precise measurements in the large-bubble regime. However, this is at the expense of the signal between 60 and 90 minutes, which cannot be precisely measured using the second configuration.
Within the first 60 minutes, the figure 3 first confirms that the variation of the phase and amplitude along a translation are much sharper than the slow drifts due to aging. Secondly, the transmission shows a minimum at 30 minutes, consistently with [14]. This is not the maximum of attenuation: the latter happens at a shorter aging time (around 17 minutes), when the variation of the amplitude over a translation $x_{+}-x_{-}$is the highest. Note also that between 20 and 40 minutes, the variation of the phase and of the amplitude become irregular, even with non-monotonous behavior along a translation. In this range, the linear fitting procedure cannot be used. More complex fitting formula can be derived, taking into account multiple reflections, but finally cannot be used here, as the range of variations of $x$ or $\log (|T|)$ are too small to provide correct fits. As a consequence, there is a range where the acoustic properties cannot be determined.

Lastly, one can see a phase jump at an aging time of 28 minutes, that survived our unwrapping procedure. This is not problematic, since we do not use the value of the offset $\operatorname{Im}[A]$ in $(6)$.

Apart from the two gaps discussed previously (for $20^{\prime}<t<40^{\prime}$ and $60^{\prime}<t<90^{\prime}$ ) where measurements cannot be done, we extract from the transmitted signal, the complex wave vector in the foam and can plot the real and imaginary parts as a function of the bubble radius (Fig. 4). For such SDS foams, the time evolution of 

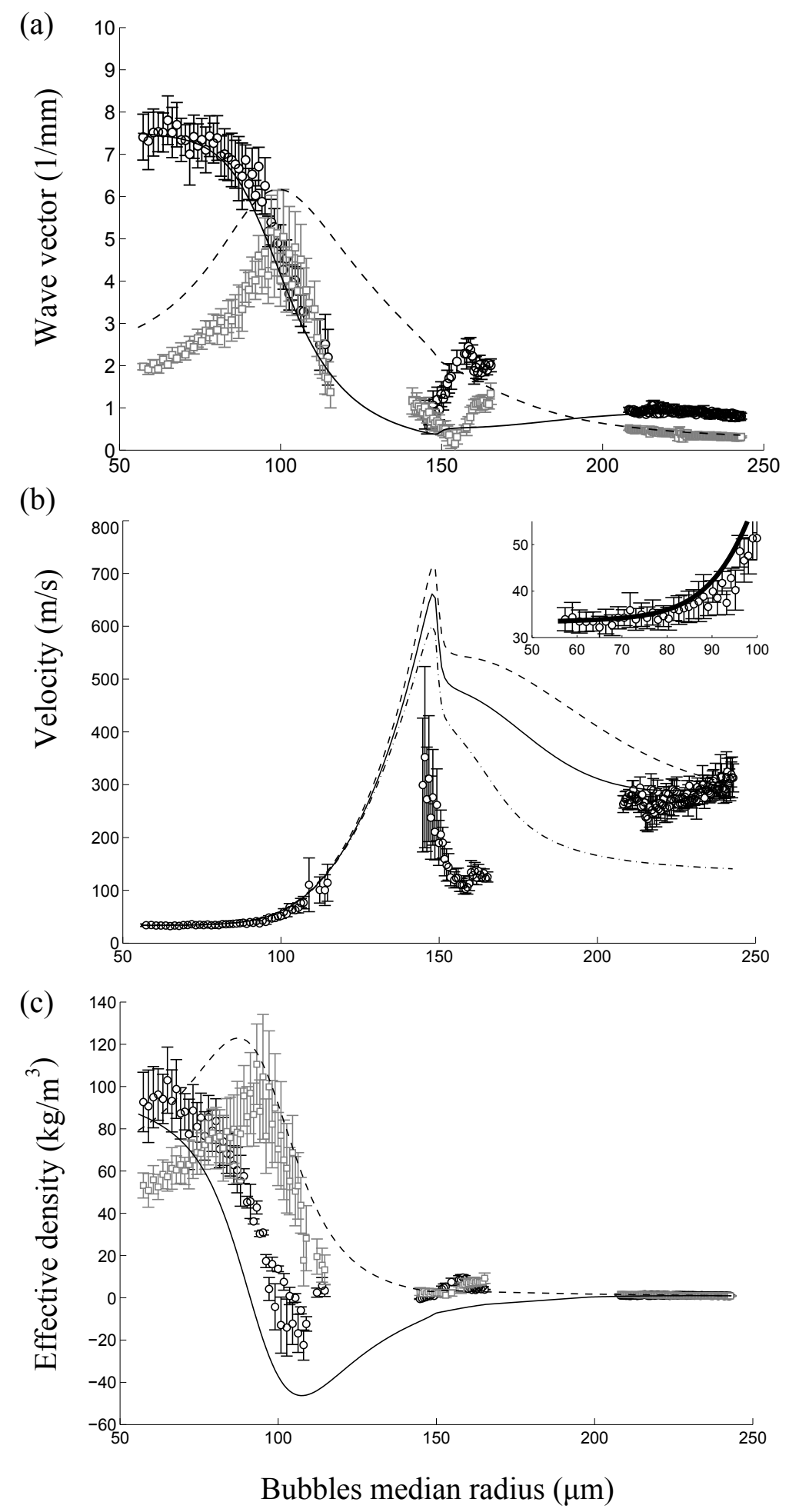

FIG. 4. Acoustic properties of the SDS foam as a function of the mean bubble radius. (a) Real (o) and imaginary ( $\square$ ) part of the acoustic wavevector, and their fits by Eq. (8), in plain curve for the real part and in dotted curve for the imaginary part. (b) Speed of sound (inset: zoom of the small radii), fitted by the model presented in Sec. V, with three different gas contents: pure $\mathrm{C}_{2} \mathrm{~F}_{6}$ (dash-dotted curve), pure air (dashed curve) and air- $\mathrm{C}_{2} \mathrm{~F}_{6}$ mixture (plain curve). (c) Real (o) and imaginary ( $\square$ ) part of the effective density, and their fits by Eq. (9), in plain curve for the real part and in dotted curve for the imaginary part. 
the bubble radius is shown in Fig. 1 and this provides the way to shift from time to bubble size variation. We also derived the phase velocity $(v=\omega / \operatorname{Re}(k))$ and the effective density, and stacked all these plots in figure 4. Figure 4 shows that the acoustic wave propagation is indeed strongly dispersive, confirming previous works [14, 18]. Under this form, the data can then be directly compared to the model of [18]. This model predicts the acoustic response for all bubble sizes, and the challenge here is to check whether there is a range of fitting parameters which can described the whole experimental behavior, both in terms of complex wave vector, velocity and complex effective density, and not only for velocities in asymptotic cases.

To compute the wavevector following the expressions presented in Sec. V and to compare it to the data, we need values for the liquid fraction $\Phi$, the gas composition, the film thickness $e$, and the viscous time $\tau$. It first comes that the data cannot be fitted with a constant liquid fraction. We thus consider a simple linear decrease of the liquid fraction as a function of $R$. As well, the gas composition also needs to be modified along the aging. All together, the optimal fitting parameters are: a liquid fraction decreasing between $13 \%$ to $6 \%$, a viscous time equal to $2 \times 10^{-5} \mathrm{~s}$, a constant film thickness equal to $250 \mathrm{~nm}$, and a gas evolving between pure $\mathrm{C}_{2} \mathrm{~F}_{6}$ at the beginning of the experiment to pure air at the end (with a linear dependence of the proportion of both gases with the bubble size). With such conditions, the predictions of the model is superposed to the data of figure 4. To illustrate the effect of the gas composition, we also show the predictions for pure $\mathrm{C}_{2} \mathrm{~F}_{6}$, and pure air.

From the comparisons on Fig. 4, it finally turns out that the model captures most of the non-monotonous experimental trends: a low speed of sound for small bubbles, a large speed of sound for large bubbles, and a resonance in between with a maximum of attenuation. Moreover, the fitting parameters are consistent with the experimental conditions. Such initial and final liquid fractions, and gas evolution are actually in full agreement with what was expected, as discussed in Sec.2. The time $\tau$ is identical to the one found in [18], and the film thickness falls within a reasonable range, though higher than expected [18].

The gas composition effect is well evidenced: explaining the acoustic features clearly requires to take into account the air entering within the foam the velocity. The curves in figure 4 clearly shows that assuming a constant pure $\mathrm{C}_{2} \mathrm{~F}_{6}$ with time is unrealistic at the end of the experiments.

Note that the speed of sound at small bubble size, as well as the position of the resonance, is much less sensitive on the gas content than at high bubble sizes. Quantitatively, we recover the Wood's velocity in this limit of small bubble sizes: with (13), one gets $v_{\text {Wood }}=31 \mathrm{~m} / \mathrm{s}$ for pure $\mathrm{C}_{2} \mathrm{~F}_{6}$. We can then consider for the following analysis that the foam is made of pure $\mathrm{C}_{2} \mathrm{~F}_{6}$ before the resonance.
In the opposite limit, at large bubble size, the speed of sound is slightly lower than that of air. Quantitatively, we recover here the other asymptotic limit (equation 15), assuming pure air within the foam. Although the final value for the speed of sound is compatible with pure air, there might still be some low fractions of $\mathrm{C}_{2} \mathrm{~F}_{6}$, and our data are not enough precise to determine accurate gas compositions. This means also that extracting absolute values of the film thickness remains risky, if one does not monitor the gas composition. We also observe a slow increase of the speed of sound in this regime, which can either due to gas composition continuing to change, or to a decrease of the ratio $e / R$ due to the continuous growth of the bubble size (with a film thickness remaining constant). For the subsequent analysis, we consider that this second effect dominates in the large bubble size regime, and simplify these issues by considering pure air within the foam at the late stages of the experiments. This will allow us to make relative comparisons of film thickness for the different formulations.

The model thus quantitatively explain the behavior in the two extreme limits. However, the agreement between the data at the model is less good in the intermediate range of bubble size associated to the resonance. It is found that the radius for the maximum of attenuation (here, at $R=100$ microns) is well recovered by the model, but the attenuation is always overestimated, as shown in Fig. 4. Also, for bubble radius between 140 and $170 \mu \mathrm{m}$, the agreement between the data and the model is poor, even if the quantitative range for the wavevector (between 1 and $2 \mathrm{~mm}^{-1}$ ) is fine. In that range of $R$, there are also discrepancies between the predictions and the data for the velocity.

It is interesting to point out that the model also gives us some insights on the range between $120 \mu \mathrm{m}$ and $140 \mu \mathrm{m}$ where no measurements were possible. This range corresponds to an effective density with a small modulus: the acoustic impedance of the foam is then very mismatched from that of the transducers, and the acoustic power is poorly transmitted between the transducers and the foam, both at the emitting and at the receiving transducers. Moreover, this range of bubble size also corresponds to a real part of the effective density becoming negative, together with the imaginary part close to zero. Such features, as already reported in [18], suggest a regime where sound does not propagate but forms an evanescent wave (although dissipation complicates this simple picture). In parallel, for these bubble sizes, the velocity is expected to get highest values. It is then clear that measuring acoustic features within this range is extremely difficult. In fact, problems to obtain clean data are associated to sizes where $\operatorname{Re}\left(\rho_{\text {eff }}\right)$ and $\operatorname{Im}\left(\rho_{\text {eff }}\right)$ are close to zero or negative, while velocity is high.

All together, the comparisons of the global model proposed in [18] to our data spanning over a large range of bubble radii shows that this model captures well the main features. This is even quantitatively correct below and above a resonance regime, for which the sound prop- 
agation is highly complex. Moreover, the superposition in Fig. 4 of the data and predictions tends to provide a complete picture, allowing us to connect the different parts of the foam acoustic behavior.

\section{INFLUENCE OF THE CHEMICAL FORMULATION ON ULTRASONIC PROPAGATION}

Following the previous detailed analysis of our reference SDS foams, we can then analyze and compare the measurements made for all the other chemical formulations listed in Tab. I.

Our first major result is that the same qualitative features are recovered whatever the foam recipes. The consecutive behaviors described in Fig. 4, as the bubble size increases, are always observed: the complex resonance regime always separates the two well-defined behaviors at small and large bubble sizes. It is especially in these regimes before and after the resonance that data can be safely compared, and that quantitative differences with the formulation are actually found.

Focussing on the evolution before the resonance, the figure 5 shows the sound velocity as a function of the bubble radius, for different type of foams. For clarity, not all the data points are plotted. As for the SDS foam, the other data sets show an asymptotic velocity at the lowest bubble sizes, followed by an rapid increase of this velocity as the resonance is approached. Note the case of SDS-glycerol foams, where the velocity increases already drastically at the smallest bubble size, so that we hardly detect the asymptotic limit. Nevertheless, this velocity plateau is found independent of the solution and the value is in agreement with the SDS foam. This tends to validate that - for any type of foams and in the limit of small bubble sizes - the sound propagation agrees with the basic assumptions of the Wood's model.

By contrast, it is also clear from figure $6 \mathrm{a}$ that the chemical formulations modify the resonant radius. We will consider three characteristic radii: the resonance radius $R^{*}$ predicted from the model of [18], Eq. (16); the radius $R^{\dagger}$ coming from the small-bubble limit, Eq. (17); and the radius of maximal attenuation, $R^{\prime}$. Using the approximated formula (17), valid for $q a \ll 1$, the data can be adjusted to extract the radius $R^{\dagger}$. The error bar for this radius comes from the nonlinear fitting procedure. In the figure $6 \mathrm{a}, R^{\dagger}$ is plotted for the different foam types. The radius $R^{\prime}$ corresponding to the maximum of attenuation (like the ones of figure 4 ) is also added. The time when the maximum of attenuation is reached is found with great accuracy; in contrast, the conversion between this time and the radius of maximal attenuation $R^{\prime}$, using Eq. (2), comes from a linear fit of the square of the mean bubble radius as a function of time (Fig. 1). It is this conversion, and the uncertainty on the latter fit, which gives the major uncertainty on $R^{\prime}$, hence its error bar. Finally, we also plot the resonance radius $R^{*}$ pre- dicted from Eq. (16); all experimental parameters in this expression are known precisely except $\Phi$, which varies between $8 \%$ and $12 \%$. It is this uncertainty which gives the error bar on $R^{*}$.

The comparisons first show that all the radii fall within the same range: the two measured values are always close to the predicted ones and despite large differences in the type of foams, there is less than a factor 3 between the extreme values. Thus, at a first order, a good agreement between all the data and the model is found, tending to show that physical parameters (like bubble size and liquid fraction) are more crucial in foam acoustics than chemical ones.

Still, some systematic deviations can be observed. The addition of $\mathrm{DOH}$ which acts only as a provider of interfacial viscoelasticity gives higher values for the radius at the resonance, when compared to the model. At some point, it might be possible that, between interfacial elasticity and surface tension, the biggest value must be taken in equation (16), as found in emulsion rheology [31]. In that sense, dilatational elasticity replaces surface tension once it becomes significantly higher.

Oppositely, increasing the bulk viscosity, by adding glycerol, gives smaller values. Smaller values are also found for the protein foams, which differ from SDS foam at the scale of the thin films (thicker and gelified). Finally, the chemical system (SDS with CP) which is expected to be the most different from the reference foam (at interfaces, in films and in bulk), gives a similar resonance radius. However, despite the same velocity plateau value and resonance radius for SDS and SDS/CP foams, the shape of the curve is different for these two systems (Fig.5). At this point, these nontrivial behaviors remain hard to explain, but they mostly show that complex chemical formulations can have only a little effect of the acoustic resonance, possibly as a consequence of combined opposite effects. One may also wonder whether the differences of resonance radii observed between different formulations is not a mere effect of a lack of reproducibility of the measurements. To address this point, we repeated the reference experiment, and the one with the formulation SDS (10 g/L)-PC (3 g/L). Fig. 6a shows then that the relative uncertainty on the resonance radii is about $5 \%$ for a given formulation, which is lower than the differences measured between different formulations.

Then, we can focus on the behavior above the resonance. Following the data analysis of Sec. VI, and considering pure air within all the foams, we have plotted the film thickness for the various foam types (figure $6 \mathrm{~b}$ ). To do so, we have used Eq. (15), and we have performed, at large radius, a linear fit of $1 / v$ as a function of $1 / R$; the error bars come from the uncertainty of the fit exactly as for $v$ and $\alpha$ (Sec. IV). Note that using Eq. (15), we have access to the product $x e$ and not only the film thickness. As discussed previously, an absolute measurement requires to know the exact gas composition. As a consequence, we prefer to compare the data in a relative way: we have thus normalized all the thicknesses by 


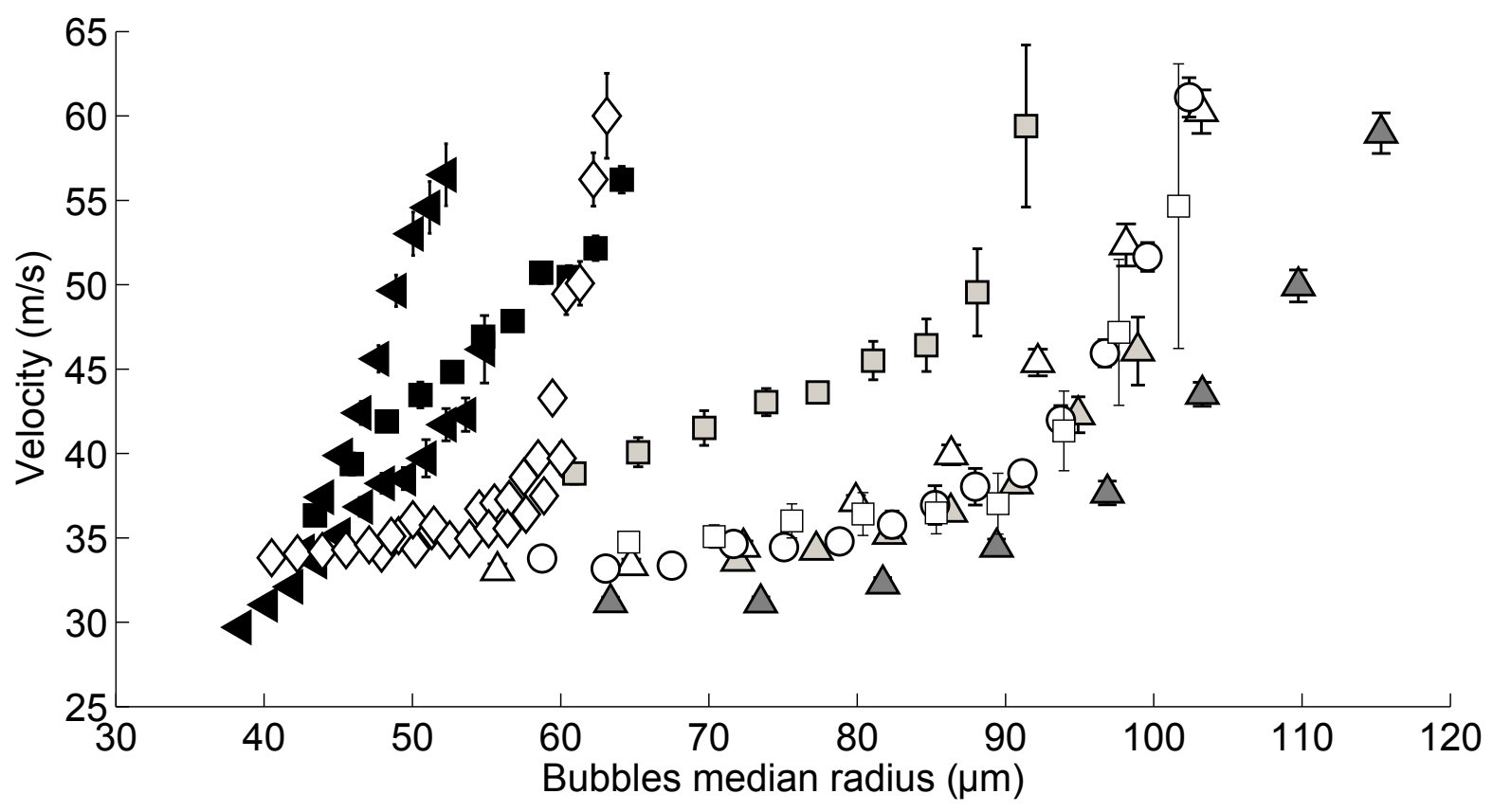

FIG. 5. Speed of sound as a function of the mean bubble radius for different formulations: SDS (o), SDS with dodecanol (upper triangles: the darker the color, the larger the concentration in dodecanol), SDS with polymer ( $\square$ : the darker the color, the larger the concentration in polymer), SDS with glycerol $(\triangleleft)$ and casein $(\diamond)$.

the average value found for the SDS foams. It turns out that the results are in agreement with the independent single film studies: the thinnest films are found for the SDS and SDS-DOH systems, while the thicker ones are observed for the polymer and protein films. Thus, all the data are correctly sorted. However, an increase by a factor 3 between the two extreme values is lower than the ratio expected from single film microscopy. From these measurements, a larger ratio is expected (from typically 70-100 $\mathrm{nm}$ for the SDS films to $500 \mathrm{~nm}$ for the thicker films). As explained before, the discrepancy might come from different gas compositions. But it might also be possible that the microscopy of the single films is done on films of millimeter dimension, and that they do not fully corresponds to those inside a foam of 100 microns bubble diameters. Furthermore, the vibrating films might be thicker than the static ones observed in microscopy, due to dynamic effects [32], especially for SDS; this could also explain the unexpected large thickness used in the model to fit SDS data. Overall, this is illustrating that there is a regime where in situ film thickness measurement could in principle be done, provided that the gas is known.

\section{CONCLUSIONS}

Sound propagation in foams - even in the case where the bubble size is small compared to the sound wavelength - turns out to be much more complex than expected; however, the recent theoretical and experimental results allows us to capture the main acoustic features and their microscopic origins.

Our data at fixed frequency as a function of the bubble size are fully in agreement with those at varied frequency and fixed bubble sizes [18]. Both type of experiments coincide on the fact that there is a shift, when varying either the frequency or the bubble size, between two limits, separated by an intermediate non-trivial propagation, including a resonance of the structure film/Plateau border and associated to an effective negative density.

The regime before the resonance corresponds to a modification of the sound propagation in the pure gas only linked to the liquid fraction. The acoustic waves are not sensitive to the foam structure. Oppositely, the difference from the pure gas above the resonance arise from a completely different origin, and linked to the film thickness. Finally, the intermediate regime (the resonance) is sensitive to the entire foam structure.

These new results are indeed qualitatively independent of the chemistry of the foaming solution. However, some quantitative differences arise when bulk and interfacial viscoelasticity are varied. But, we show here that these differences remain small. Moreover, it seems that the existing model, based on the physical parameters of a foam, already well capture the experimental results, so that interfacial and bulk rheological properties does not seem to be the main key parameters controlling the foam acoustics. In parallel, the data analysis also evidences the important role of the gas composition, especially in the regime after the resonance. 
(a)

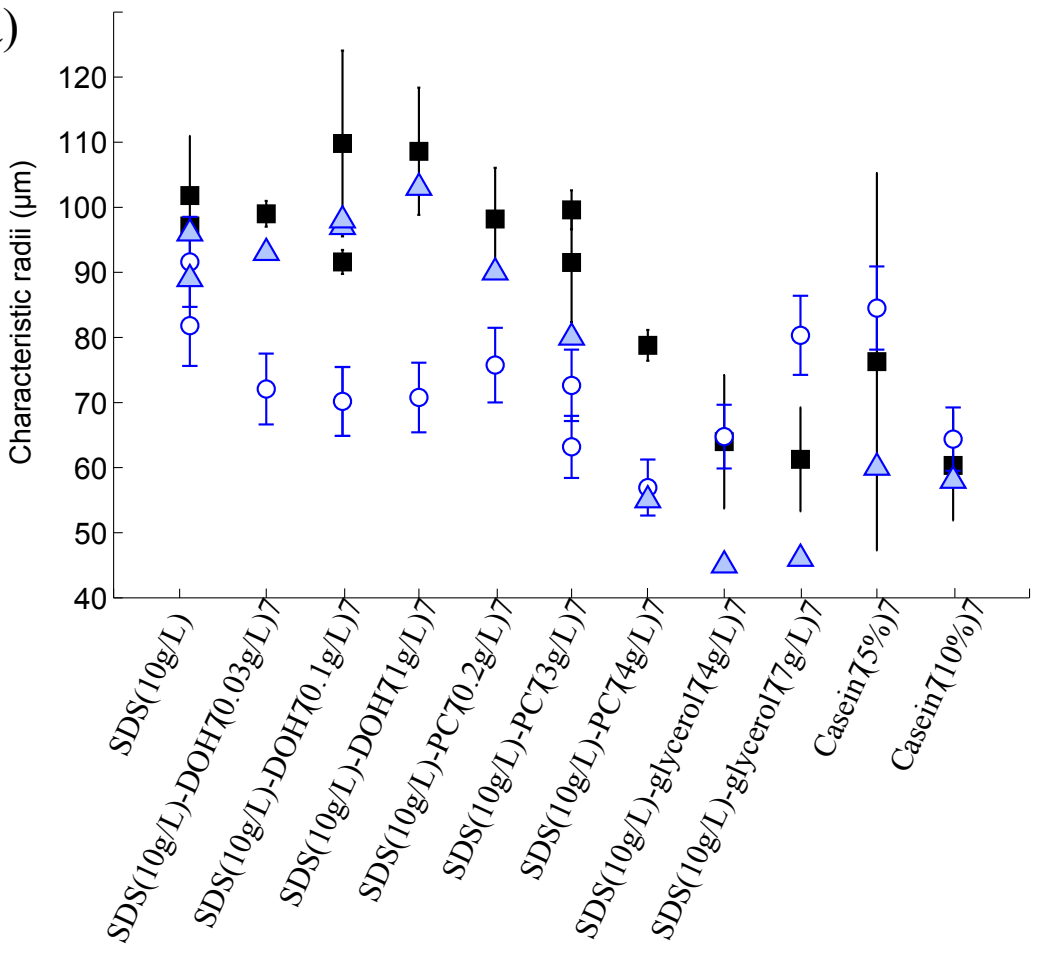

(b)

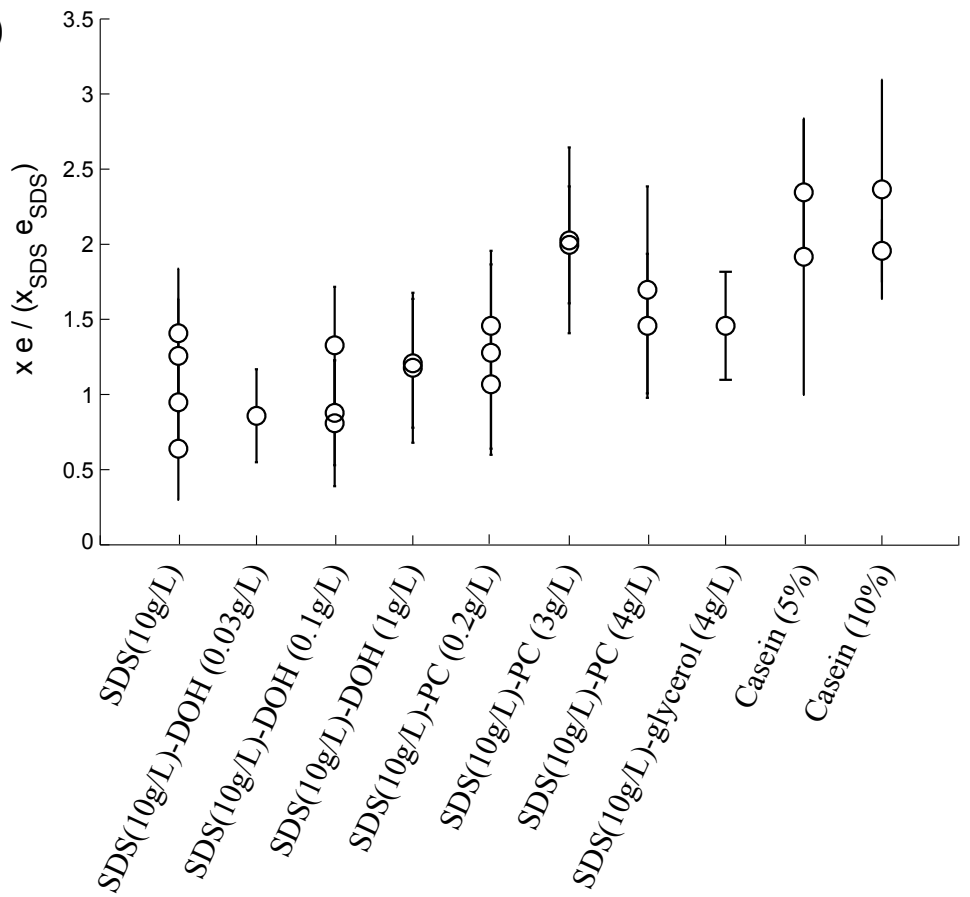

FIG. 6. (Color online) (a) Characteristic radii for the different types of foam. $\square$ : Maximum of the attenuation $R^{\prime} . \triangle$ : Radius $R^{\dagger}$ extracted from the fit at small bubble size, Eq. (17). $\bigcirc$ : radius of resonance $R^{*}$ from equation 16 for $\Phi=[0.08-0.12]$. (b) Relative thicknesses $e$ for the different foams. 
Overall, even if some data are still lacking (intermediate sizes, gas content), our study yields a consistent picture to the acoustics of foams, implementing the results of $[14,18]$, and extending it to different chemical formulations.

We also show here that such simple measurements could be first used to monitor the liquid fraction, and provide absolute measurement of $\Phi$, without the need of any calibration. Secondly, one could also use such setup to get in situ film thickness, which remain a never done measurement within foams of bubble sizes smaller than millimeters. But any absolute measurements of film thickness will require to know the exact gas composition. This also opens an interesting perspective: in previous experimental studies [27], different gases have been used to tune the coarsening rate, but gas has never been shown to have a such significant effect on macroscopic foam properties (optics, conductimetry or rheology). The fact that it influences significantly the acoustical properties of the foams thus calls for further studies of the coarsening of foams constituted by mixture of gases.

\section{ACKNOWLEDGMENTS}

We acknowledge funding support from Agence Nationale de la Recherche in the frame of the SAMOUSSE project (ANR-11-BS09-001). We thank all the participants of this project, especially Valentin Leroy, for fruitful discussions.
[1] R. K. Prud'homme and S. A. Khan, Foams: Theory, Measurements, and Applications (Marcel Dekker Inc., New York, 1997).

[2] D. Weaire and S. Hutzler, The Physics of Foams (Oxford University Press, New York, 1999).

[3] I. Cantat, S. Cohen-Addad, F. Elias, F. Graner, R. Höhler, O. Pitois, F. Rouyer, and A. Saint-Jalmes, Foams - Structure and Dynamics, 1st ed., edited by S. J. Cox (Oxford University Press, 2013).

[4] R. Höhler, S. Cohen-Addad, and D. J. Durian, Curr. Opin. Colloid Interface Sci. 19, 242 (2014).

[5] K. Feitosa, S. Marze, A. Saint-Jalmes, and D. J. Durian, J. Phys. Condens. Matter 17, 6301 (2005).

[6] D. A. Weitz and D. J. Pine, Dynamic Light Scattering: the Methods and Applications (Oxford University Press, Oxford, 1993).

[7] D. J. Durian, D. A. Weitz, and D. J. Pine, Phys. Rev. A 44, R7902 (1991).

[8] M. U. Vera, A. Saint-Jalmes, and D. J. Durian, Appl. Opt. 40, 4210 (2001).

[9] I. Goldfarb, Z. Orenbach, I. Schreiber, and F. Vafina, Shock Waves 7, 77 (1997).

[10] N. Mujica and S. Fauve, Phys. Rev. E 66, 021404 (2002).

[11] K. B. Kann, Colloids Surf. A 263, 315 (2005).

[12] A. Britan, M. Liverts, and G. Ben-Dor, Colloids Surf. A 344, 48 (2009).

[13] M. Erpelding, R.-M. Guillermic, B. Dollet, A. SaintJalmes, and J. Crassous, Phys. Rev. E 82, 021409 (2010).

[14] I. Ben Salem, R.-M. Guillermic, C. Sample, V. Leroy, A. Saint-Jalmes, and B. Dollet, Soft Matter 9, 1194 (2013).

[15] J. Pierre, F. Elias, and V. Leroy, Ultrasonics 53, 622 (2013).
[16] J. Pierre, R. Guillermic, F. Elias, W. Drenckhan, and V. Leroy, Eur. Phys. J. E 36, 113 (2013).

[17] J. Pierre, V. Leroy, A. Saint-Jalmes, B. Dollet, I. Ben Salem, J. Crassous, R.-M. Guillermic, W. Drenckhan, and F. Elias, Proc. Meetings Acoust. 19, 045044 (2013).

[18] J. Pierre, B. Dollet, and V. Leroy, Phys. Rev. Lett. 112, 148307 (2014).

[19] M. Karbaschi, M. Lofti, J. Kragel, A. Javadi, D. Bastani, and R. Miller, Curr. Opin. Colloid Interface Sci. 19, 514 (2014).

[20] L. M. C. Sagis and P. Fischer, curr. Opin. Colloid Interface Sci. 19, 520 (2014).

[21] H. A. Barnes, J. F. Hutton, and K. Walters, An Introduction to Rheology (Elsevier, Amsterdam, 1989).

[22] K. J. Mysels, K. Shinoda, and S. Frenkel, Soap Films, Studies of Their Thinning and a Bibliography (Pergamon Press, London, 1959).

[23] V. Bergeron, J. Phys. Condens. Matter 11, R215 (1999).

[24] C. Stubenrauch and R. von Klitzing, J. Phys. Condens. Matter 15, R1197 (2003).

[25] E. Dickinson, Soft Matter 2, 642 (2006).

[26] G. Maurdev, A. Saint-Jalmes, and D. Langevin, J. Colloid Interface Sci. 300, 735 (2006).

[27] A. Saint-Jalmes, Soft Matter 2, 836 (2006).

[28] S. A. Koehler, S. Hilgenfeldt, and H. A. Stone, Langmuir 16, 6327 (2000).

[29] H. M. Princen, J. Colloid Interface Sci. 105, 150 (1985).

[30] A. B. Wood, A Textbook of Sound (Bell and Sons, London, 1944).

[31] T. D. Dimitrova and F. Leal-Calderón, Adv. Colloid Interface Sci. 108, 49 (2004).

[32] F. Elias, S. Hutzler, and M. S. Ferreira, Eur. J. Phys. 28, 755 (2007). 